\title{
Generation of GW Radiation Pulses from a VUV Free-Electron Laser Operating in the Femtosecond Regime
}

V. Ayvazyan, ${ }^{4}$ N. Baboi, ${ }^{7,16}$ I. Bohnet,${ }^{5}$ R. Brinkmann, ${ }^{4}$ M. Castellano, ${ }^{8}$ P. Castro, ${ }^{4}$ L. Catani, ${ }^{10}$ S. Choroba, ${ }^{4}$ A. Cianchi, ${ }^{10}$ M. Dohlus,${ }^{4}$ H. T. Edwards, ${ }^{6}$ B. Faatz ${ }^{4}$ A. A. Fateev, ${ }^{13}$ J. Feldhaus, ${ }^{4}$ K. Flöttmann, ${ }^{4}$ A. Gamp, ${ }^{4}$ T. Garvey, ${ }^{14}$ H. Genz, ${ }^{3}$ Ch. Gerth, ${ }^{4}$ V. Gretchko, ${ }^{11}$ B. Grigoryan, ${ }^{19}$ U. Hahn, ${ }^{4}$ C. Hessler, ${ }^{3}$ K. Honkavaara, ${ }^{4}$ M. Hüning, ${ }^{17}$ R. Ischebeck, ${ }^{17}$ M. Jablonka, ${ }^{1}$ T. Kamps, ${ }^{5}$ M. Körfer, ${ }^{4}$ M. Krassilnikov, ${ }^{2}$ J. Krzywinski, ${ }^{12}$ M. Liepe, ${ }^{7}$ A. Liero, ${ }^{17}$ T. Limberg, ${ }^{4}$ H. Loos, ${ }^{3}$ M. Luong, ${ }^{1}$ C. Magne, ${ }^{1}$ J. Menzel, ${ }^{17}$ P. Michelato, ${ }^{9}$ M. Minty, ${ }^{4}$ U.-C. Müller, ${ }^{4}$ D. Nölle, ${ }^{4}$ A. Novokhatski, ${ }^{2}$ C. Pagani, ${ }^{9}$ F. Peters, ${ }^{4}$ J. Pflüger, ${ }^{4}$ P. Piot, ${ }^{4}$ L. Plucinski, ${ }^{7}$ K. Rehlich, ${ }^{4}$ I. Reyzl, ${ }^{4}$ A. Richter, ${ }^{3}$ J. Rossbach, ${ }^{4}$ E. L. Saldin, ${ }^{4}$ W. Sandner, ${ }^{15}$ H. Schlarb, ${ }^{7}$ G. Schmidt, ${ }^{4}$ P. Schmüser, ${ }^{7}$ J. R. Schneider, ${ }^{4}$ E. A. Schneidmiller, ${ }^{4}$ H.-J. Schreiber, ${ }^{5}$ S. Schreiber, ${ }^{4}$ D. Sertore, ${ }^{9}$ S. Setzer, ${ }^{2}$ S. Simrock, ${ }^{4}$ R. Sobierajski, ${ }^{4,18}$ B. Sonntag, ${ }^{7}$ B. Steeg, ${ }^{4}$ F. Stephan, ${ }^{5}$ K. P. Sytchev, ${ }^{13}$ K. Tiedtke, ${ }^{4}$ M. Tonutti, ${ }^{17}$ R. Treusch, ${ }^{4}$ D. Trines, ${ }^{4}$ D. Türke, ${ }^{17}$ V. Verzilov, ${ }^{8}$ R. Wanzenberg, ${ }^{4}$ T. Weiland, ${ }^{2}$ H. Weise, ${ }^{4}$ M. Wendt,${ }^{4}$ I. Will, ${ }^{15}$ S. Wolff, ${ }^{4}$ K. Wittenburg, ${ }^{4}$ M. V. Yurkov, ${ }^{13, *}$ and K. Zapfe ${ }^{4}$

${ }^{1}$ CEA Saclay, 91191 Gif sur Yvette, France

${ }^{2}$ Darmstadt University of Technology, FB18-Fachgebiet TEMF, Schlossgartenstrasse 8, 64289 Darmstadt, Germany

${ }^{3}$ Darmstadt University of Technology, Institut für Kernphysik, Schlossgartenstrasse 9, 64289 Darmstadt, Germany

${ }^{4}$ Deutsches Elektronen-Synchrotron DESY, Notkestrasse 85, 22603 Hamburg, Germany

${ }^{5}$ Deutsches Elektronen-Synchrotron DESY, Platanenallee 6, 15738 Zeuthen, Germany

${ }^{6}$ Fermi National Accelerator Laboratory, MS 306, P.O. Box 500, Batavia, Illinois 60510

${ }^{7}$ Hamburg University, Institut für Experimentalphysik, Notkestrasse 85, 20603 Hamburg, Germany

${ }^{8}$ INFN-LNF, via Enrico Fermi 40, 00044 Frascati, Italy

${ }^{9}$ INFN Milano-LASA, via Fratelli Cervi 201, 20090 Segrate, Milano, Italy

${ }^{10}$ INFN-Roma2, via della Ricerca Scientifica 1, 00100 Roma, Italy

${ }^{11}$ Institute for Nuclear Research of RAS, 117312 Moscow, 60th October Anniversary prospect 7A, Russia

${ }^{12}$ Institute of Physics, Polish Academy of Sciences, al. Lotnikow, 32/46, 02-668 Warsaw, Poland

${ }^{13}$ Joint Institute for Nuclear Research, 141980 Dubna, Moscow Region, Russia

${ }^{14}$ Laboratoire de l'Accélérateur Linéaire, IN2P3-CNRS, Université de Paris-Sud, BP 34, F-91898 Orsay, France

${ }^{15}$ Max-Born-Institute, Max-Born Strasse 2a, 12489 Berlin, Germany

${ }^{16}$ NILPRP, P.O. Box MG-36, Bucharest, Romania

${ }^{17}$ RWTH Aachen-Physikzentrum, Physikalischen Institutes IIIa, Sommerfeldstrasse 26-28, 52056 Aachen, Germany

${ }^{18}$ Warsaw University of Technology, Institute of Physics, Poland

${ }^{19}$ Yerevan Physics Institute, 2 Alikhanyan Brothers strasse, 375036 Yerevan, Armenia

(Received 18 December 2001; published 25 February 2002)

\begin{abstract}
Experimental results are presented from vacuum-ultraviolet free-electron laser (FEL) operating in the self-amplified spontaneous emission (SASE) mode. The generation of ultrashort radiation pulses became possible due to specific tailoring of the bunch charge distribution. A complete characterization of the linear and nonlinear modes of the SASE FEL operation was performed. At saturation the FEL produces ultrashort pulses (30-100 fs FWHM) with a peak radiation power in the GW level and with full transverse coherence. The wavelength was tuned in the range of $95-105 \mathrm{~nm}$.
\end{abstract}

PACS numbers: 41.60.Cr, 29.17.+w, 41.75.Lx

Future progress in the synchrotron radiation studies is connected with the progress in development of fourth generation synchrotron radiation sources which is related to the progress of Self-Amplified Spontaneous emission Free-Electron Lasers (SASE FELs). The free electron laser at the TESLA Test Facility (TTF) at the Deutsches Elektronen-Synchrotron DESY [1-3] produces radiation during a single pass of electron beam through a long undulator [4-6]. The amplification process in the SASE FEL develops from shot noise in the electron beam, and intense, coherent radiation is produced in a narrow band around the resonance wavelength. In the high-gain linear regime, the radiation power $P(z)$ grows exponentially with the distance $z$ along the undulator [7-9]: $P(z) \propto \exp \left(z / L_{\mathrm{g}}\right)$, where $L_{\mathrm{g}}$ is the power gain length. Since our first report on lasing [10], the performance of the SASE FEL improved steadily. The radiation wavelength is continuously tunable in a wide range from 80 to $180 \mathrm{~nm}$ [11], and recently saturation has been achieved [12].

A detailed description of the experimental facility was presented in [10-12]. The main parameters for the FEL operation are compiled in Table I. The TTF FEL consists of a linear electron accelerator producing bunches with an energy up to $300 \mathrm{MeV}$ and a $14.1 \mathrm{~m}$ long undulator section [13]. The injector is a laser-driven radio frequency (rf) gun [14-16], followed by a superconducting cavity which 
TABLE I. Main parameters of the TESLA Test Facility for FEL experiments (TTF FEL, phase 1).

\begin{tabular}{lc}
\hline \hline Beam energy & $240-250 \mathrm{MeV}$ \\
Bunch charge & $2.7-3.3 \mathrm{nC}$ \\
Charge in radiative part of bunch & $0.1-0.2 \mathrm{nC}$ \\
Peak current & $1.3 \pm 0.3 \mathrm{kA}$ \\
rms energy spread & $150 \pm 50 \mathrm{keV}$ \\
rms normalized emittance & $(6 \pm 2) \pi \mathrm{mrad} \cdot \mathrm{mm}$ \\
Bunch spacing & $0.44 / 1 \mu \mathrm{s}$ \\
Number of bunches in a train & up to 70 \\
rf pulse repetition rate & $1 \mathrm{~Hz}$ \\
Undulator period $\lambda_{u}$ & $2.73 \mathrm{~cm}$ \\
Undulator peak field & $0.47 \mathrm{~T}$ \\
Average beta function & $1.2 \mathrm{~m}$ \\
Magnetic length of undulator & $13.5 \mathrm{~m}$ \\
Radiation wavelength & $95-105 \mathrm{~nm}$ \\
Energy in the radiation pulse & $30-100 \mu \mathrm{J}$ \\
FWHM radiation pulse duration & $50+50 \mathrm{fs}$ \\
Radiation peak power level & $1 \mathrm{GW}$ \\
Radiation average power up to & $5 \mathrm{~mW}$ \\
Spectrum width (FWHM) & $1 \%$ \\
Spot size at undulator exit (FWHM) & $250 \mu \mathrm{m}$ \\
Angular divergence (FWHM) & $260 \mu \mathrm{rad}$ \\
\hline \hline
\end{tabular}

boosts the energy to $16 \mathrm{MeV}$. At $3 \mathrm{nC}$ bunch charge, the longitudinal charge density distribution is approximately Gaussian with $4 \mathrm{~mm}$ rms length. The main accelerator contains two superconducting acceleration modules [17] separated by a magnetic bunch compressor [18].

As a consequence of the fairly large bunch length in comparison with the rf wavelength of $23 \mathrm{~cm}$, the particle distribution in the longitudinal phase space acquires an rf-induced curvature during the acceleration in the first module. Downstream of the bunch compressor, the charge distribution is strongly non-Gaussian with a narrow leading peak and a long tail (see Fig. 1). The estimated peak current of $1-1.5 \mathrm{kA}$ is consistent with the peak current inferred from the analysis of the FEL radiation data (see below).

For a characterization of the FEL process, the energy of the radiation as well as its spectral characteristics, angular distribution, and statistical properties have been measured. Figure 2 presents the average energy in the radiation pulse as a function of the active undulator length, defined as the distance over which the electron beam and the photon beam overlap. The active length can be varied over a large range by generating suitable orbit displacements of the electron beam, making use of the steering dipole magnets mounted inside the undulator. The orbit kick produced by a steerer is sufficient to inhibit the FEL amplification process downstream of this dipole. The energy in the radiation pulse has been measured by means of a detector, located $12 \mathrm{~m}$ downstream of the undulator. It consists of a gold wire mesh scattering light onto a microchannel plate (MCP) [20]. The dynamic range of such a detector is about 7 orders of magnitude, thus overlapping the entire operating range of the SASE FEL intensities. An independent

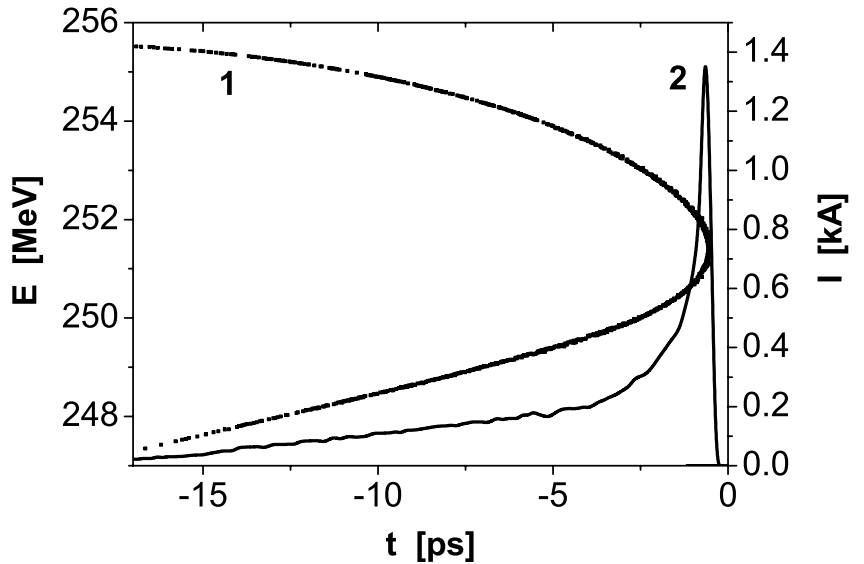

FIG. 1. Simulation of the longitudinal phase space distribution of the electrons (1) and the distribution of the current along the bunch (2) at the undulator entrance, taking into account the rf fields applied for acceleration, Coulomb space charge forces, and the coherent synchrotron radiation generated in the bunch compressor. The head of the bunch is on the right side. The total bunch charge is $3 \mathrm{nC}$.

measurement of the radiation energy at saturation with a thermopile [21] differed from the MCP result by less than $50 \%$. When the electron and the photon beam have no overlap along the whole undulator, the FEL interaction is suppressed and the detector shows the expected level of spontaneous emission of about $2.5 \mathrm{~nJ}$ (in $10 \mathrm{~mm}$ diameter aperture), collected from the full undulator length. By providing a good overlap of the two beams over an increasing length of the undulator, the FEL interaction is gradually switched on and the radiation energy rises exponentially until a plateau is reached at a level of 30 to $100 \mu \mathrm{J}$, depending on the accelerator tuning. One can wonder that the measured gain (see Fig. 2) is about $10^{4}$ which is less than that described in the literature (see, e.g., [9]). This is due to experimental conditions. FEL gain is defined as a ratio of output power to the power of effective shot noise

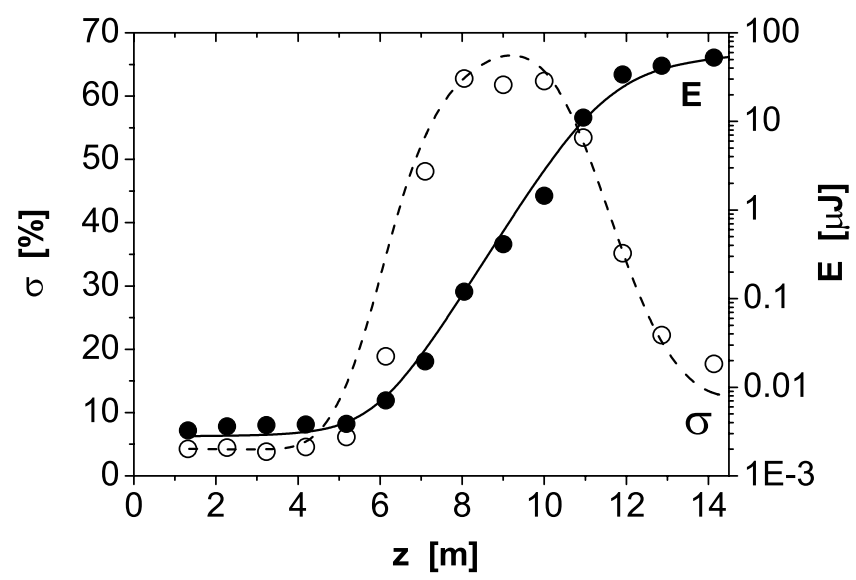

FIG. 2. Average energy in the radiation pulse (solid circles) and the rms energy fluctuations in the radiation pulse (empty circles) as a function of the active undulator length. The wavelength is $98 \mathrm{~nm}$. Circles: experimental results. Curves: numerical simulations with the code FAST [19], using parameters of Table I. 
signal [9]. The latter quantity is roughly a fraction of spontaneous radiation emitted by the electron beam at one gain length into the coherent angle. In our case, angular acceptance of the detector is 3 times larger than the coherent angle. Next, the detector always measures spontaneous radiation power emitted from the total undulator length. Finally, only $10 \%$ of the bunch charge produces coherent radiation (see Fig. 1). As a result, we come to the conclusion that a measured gain of $10^{4}$ corresponds to the FEL gain of about $10^{7}$ in terms of effective power of shot noise.

Each point in Fig. 2 represents the average over 100 bunches. The radiation pulse energy fluctuates from bunch to bunch. The rms spread $\sigma$ is plotted in Fig. 2 versus the active undulator length. For a length $z<5 \mathrm{~m}$, the rms fluctuation is on the order of $4 \%$ and mainly given by the radiation detector. When the FEL radiation exceeds the level of spontaneous emission, the rms energy spread increases rapidly to more than $60 \%$. It is essential to realize that these fluctuations are inherent to the SASE process, and not due to unstable operation of the accelerator. During the experiment, we performed simultaneous measurements of individual bunch charges and offsets at the undulator entrance, the most critical beam parameters influencing the FEL process. In order to exclude machine fluctuations, we performed selection of the pulses having fluctuations of the charge less than 1\% (rms) and orbit deviation less than $50 \mu \mathrm{m}$ (rms). After such a data selection, the measured fluctuations of the radiation energy in the bunch are dominated by the statistical properties of the SASE FEL radiation originating from shot noise in the electron beam which causes fluctuations of the beam density, which are random in time and space. The number of longitudinal optical modes amplified by the FEL is thus a statistical quantity [22]. If the average of this number (denoted $M$ in the following) is small, significant fluctuations of the output energy are to be expected. Finally, a sharp drop in the energy fluctuations is observed when the output power approaches the saturation level. This observation is a clear confirmation of the FEL saturation.

Spectral measurements are presented in Fig. 3. Singleshot spectra were taken with a monochromator $(0.2 \mathrm{~nm}$ resolution) equipped with an intensified CCD camera [21]. The bold curve represents the spectrum averaged over 100 bunches. Typical single-shot spectra consist of approximately two spikes corresponding to the value of $M$ between 2 and 3 which is in agreement with fluctuation measurements in the high-gain linear regime, giving the value of $M=2.5$. Figure 4 shows the angular divergence of the FEL radiation, obtained by scanning a $0.5 \mathrm{~mm}$ aperture mounted in front of the detector.

The information contained in Figs. 2 and 3 is sufficient to determine the SASE FEL parameters. We start with an analysis of the measured power gain length and fluctuations of the energy in the radiation pulse (see Fig. 2). From the exponential part of the gain curve, the power gain length is determined to be $L_{\mathrm{g}}=67 \pm 5 \mathrm{~cm}$. For an FEL amplifier operating in the high-gain linear regime (i.e.,

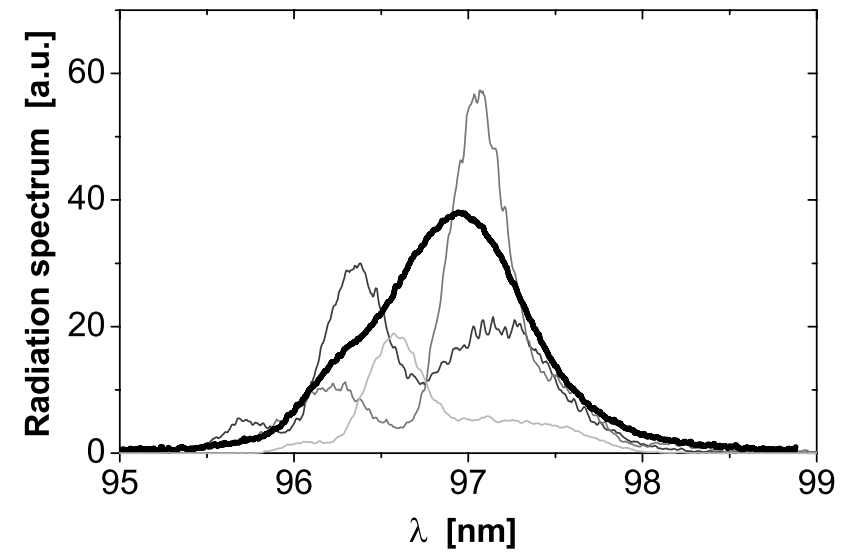

FIG. 3. Spectrum of the radiation (experimental results). The thin curves are single-shot spectra. The bold curve represents an averaged spectrum.

the exponential growth region), a parameter with a direct physical interpretation can be derived from the rms energy fluctuation $\sigma: M=1 / \sigma^{2}$ represents the number of spikes (wave packets) in the radiation pulse. The present case yields $M \simeq 2.5$ (see Fig. 2). This allows us to estimate the radiation pulse length as $\tau_{\text {rad }} \simeq M L_{\mathrm{c}} / c$ where the cooperation length is $L_{\mathrm{c}} \simeq 2 \lambda L_{\mathrm{g}} / \lambda_{\mathrm{u}} \simeq 5 \mu \mathrm{m}$. Using these numbers, the duration of the radiation pulse is found to be about $50 \mathrm{fs}$, which implies that the FEL radiation is not generated by the whole electron bunch but only by its sharp leading peak.

The FWHM width of the averaged spectrum $(\Delta \omega)_{\mathrm{FWHM}}$ is determined by the coherence length of the optical wave packets. Thus, its combination with the number of modes $M$ provides an estimate of the radiation pulse length according to [9] $\tau_{\text {rad }} \simeq 2 M \sqrt{\pi} /(\Delta \omega)_{\mathrm{FWHM}} \simeq 50$ fs. The pulse length may be independently obtained from the typical width $\Delta \omega$ of the spikes in the single-shot spectra, using the relation $\tau_{\text {rad }} \simeq 2 \pi / \Delta \omega \simeq 50 \mathrm{fs}$. It is well known that the relative FWHM spectral width, $(\Delta \omega / \omega)_{\text {FWHM }}$, corresponds approximately to $2 \rho$, where $\rho$ is the FEL parameter [6]. From the averaged spectrum (see Fig. 3), one obtains

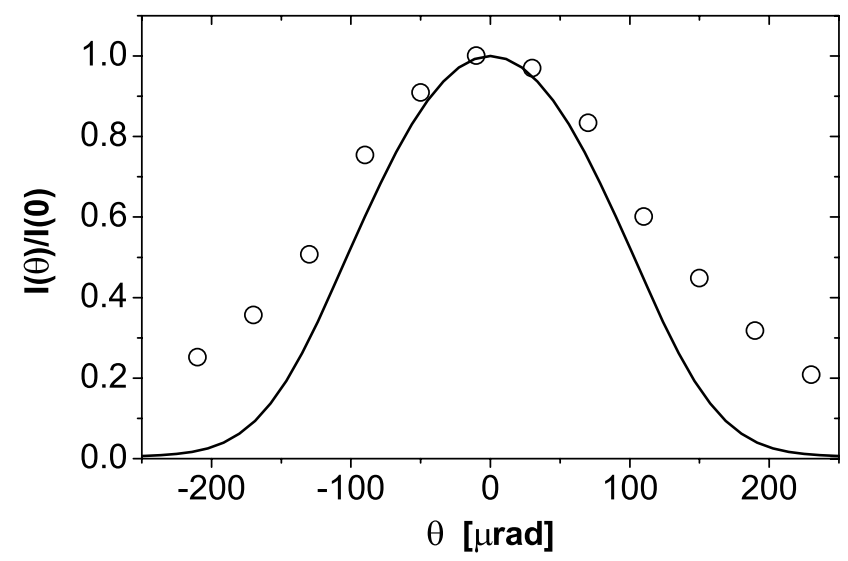

FIG. 4. Angular distribution of the radiation intensity at the undulator exit. Circles: experimental results. Solid curve: numerical simulations with the code FAST. 


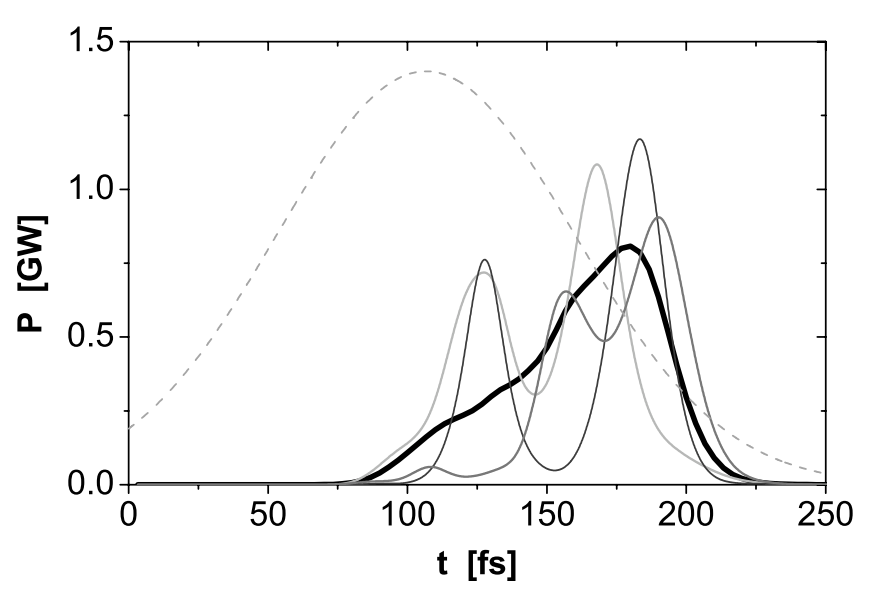

FIG. 5. Temporal structure of the radiation pulse calculated with the code FAST. The thin curves are single-shot pulses while the bold curve represents the average (FWHM pulse duration is $50 \mathrm{fs}$ ). Dashed curve: approximation of the current distribution of the head of the electron bunch by a Gaussian (see Fig. 1).

$\rho \simeq 4 \times 10^{-3}$. From the radiation pulse energy $E_{\mathrm{rad}}$, the electron energy $\mathcal{E}$, and the relation $E_{\mathrm{rad}} \simeq \rho I \mathcal{E} \tau_{\mathrm{rad}} / e$ [9], the peak current is found to be $I \simeq 1 \mathrm{kA}$, in good agreement with the bunch shape presented in Fig. 1.

A thorough analysis of the SASE FEL process can be done only by numerical simulation. For this purpose, the three-dimensional, time-dependent FEL code FAST [19] has been used. The simulation of electron bunch formation (see Fig. 1) shows that only the narrow head of the bunch is capable of generating FEL radiation. In the FEL simulations, this "radiative part" of the bunch was approximated by a Gaussian. A parameter set for this part of the electron bunch leading to simulation results consistent with our measurements is given by a FWHM duration of $120 \mathrm{fs}$, peak current $I \simeq 1.3 \mathrm{kA}$, rms energy spread $100 \mathrm{keV}$, and rms normalized emittance $\epsilon_{\mathrm{n}} \simeq 6 \pi \mathrm{mm} \cdot \mathrm{mrad}$. The simulation results (calculated from 250 statistically independent runs) are included in the plots for comparison with the experimental results (see Figs. 2-4). The good agreement between experimental data and simulation results allows us to determine the parameters of the FEL which are not directly accessible to measurement. First of all, this refers to the temporal structure of the radiation pulse (see Fig. 5). Simulations show that the computed FWHM pulse duration is about $50 \mathrm{fs}$, and the peak power is in the GW range. The spot size of the radiation beam is $250 \mu \mathrm{m}$ (FWHM) and about similar to the diameter of the electron beam. Combining this with the measured angular divergence of about $260 \mu \mathrm{rad}$ FWHM we conclude that, within the accuracy of the experiment, the FEL radiation has full transverse coherence.

Experimental observations and analysis presented in the paper are convincing to announce successful operation of a free electron laser producing femtosecond, coherent VUV radiation pulses at a GW level of peak power with a peak brilliance of about $2 \times 10^{28}$ photons $/\left[\mathrm{sec} \cdot \mathrm{mrad}^{2}\right.$. $\mathrm{mm}^{2} \cdot(0.1 \%$ bandwidth $\left.)\right]$, which is by 8 orders of mag- nitude higher than that of state of the art third generation synchrotron radiation sources. During the experiment the TTF FEL was operated at a rate of up to 70 pulses per second with an average radiation power of up to $5 \mathrm{~mW}$. Even with such a low repetition rate, the average brilliance is $10^{17}$ photons $/\left[\mathrm{sec} \cdot \mathrm{mrad}^{2} \cdot \mathrm{mm}^{2} \cdot(0.1 \%\right.$ bandwidth $\left.)\right]$.

The authors are indebted to invaluable support by the technical staff of the participating groups from the TESLA collaboration, consisting of 44 institutes from 10 countries [23].

*Corresponding author.

Email address: yurkov@sunse.jinr.ru(M.V.Yurkov)

[1] W. Brefeld et al., Nucl. Instrum. Methods Phys. Res., Sect. A 393, 119 (1997).

[2] T Åberg et al., A VUV FEL at the TESLA Test Facility at DESY, Conceptual Design Report, DESY Print TESLA-FEL 95-03, 1995.

[3] J. Rossbach, Nucl. Instrum. Methods Phys. Res., Sect. A 375, 269 (1996).

[4] A. M. Kondratenko and E. L. Saldin, Part. Accel. 10, 207 (1980).

[5] Ya. S. Derbenev, A. M. Kondratenko, and E. L. Saldin, Nucl. Instrum. Methods Phys. Res. 193, 415 (1982).

[6] R. Bonifacio, C. Pellegrini, and L. M. Narducci, Opt. Commun. 50, 373 (1984).

[7] K. J. Kim, Phys. Rev. Lett. 57, 1871 (1986).

[8] S. Krinsky and L. H. Yu, Phys. Rev. A 35, 3406 (1987).

[9] E. L. Saldin, E. A. Schneidmiller, and M. V. Yurkov, The Physics of Free Electron Lasers (Springer-Verlag, Berlin, 1999).

[10] J. Andruszkow et al., Phys. Rev. Lett. 85, 3825 (2000).

[11] J. Rossbach, presentation at FEL2000 Conference [Nucl. Instrum. Methods Phys. Res., Sect. A (to be published)].

[12] V. Ayvazyan et al. (to be published).

[13] J. Pflüger, Nucl. Instrum. Methods Phys. Res., Sect. A 445, 366 (2000).

[14] J.-P. Carneiro et al., in Proceedings of the 1999 Particle Accelerator Conference, New York (IEEE, Piscataway, NJ, 1999), pp. 2027-2029.

[15] P. Michelato et al., Nucl. Instrum. Methods Phys. Res., Sect. A 445, 422 (2000).

[16] I. Will, S. Schreiber, A. Liero, and W. Sandner, Nucl. Instrum. Methods Phys. Res., Sect. A. 445, 427 (2000).

[17] H. Weise, in Proceedings of the 1998 Linac Conference, Chicago (Argonne National Laboratory, Argonne, IL, 1998), pp. 674-678.

[18] T. Limberg et al., Nucl. Instrum. Methods Phys. Res., Sect. A 375, 322 (1996).

[19] E. L. Saldin, E. A. Schneidmiller, and M. V. Yurkov, Nucl. Instrum. Methods Phys. Res., Sect. A 429, 233 (1999).

[20] B. Faatz et al., DESY Print TESLA-FEL 2001-09, pp. $62-67$.

[21] R. Treusch et al., Nucl. Instrum. Methods Phys. Res., Sect. A 467-468, 30 (2001).

[22] E. L. Saldin, E. A. Schneidmiller, and M. V. Yurkov, Opt. Commun. 148, 383 (1998).

[23] Electronic address: http://tesla.desy.de 